Research Article

\title{
Performance Analysis of an Environmental Adaptive CFAR Detector
}

\author{
Sung Won Hong and Dong Seog Han \\ School of Electronics Engineering, Kyungpook National University, 80 Daehak-ro, Buk-gu, Daegu 702-701, Republic of Korea \\ Correspondence should be addressed to Dong Seog Han; dshan@knu.ac.kr
}

Received 25 February 2014; Accepted 26 April 2014; Published 14 May 2014

Academic Editor: Massimo Scalia

Copyright (C) 2014 S. W. Hong and D. S. Han. This is an open access article distributed under the Creative Commons Attribution License, which permits unrestricted use, distribution, and reproduction in any medium, provided the original work is properly cited.

The constant false alarm rate (CFAR) is a detection algorithm that is generally used in radar or sonar systems, but its performance depends greatly on the environment. This means that the detection performance cannot be satisfied with only a single CFAR detector. This paper evaluates mathematically a proposed environmental adaptive (EA) CFAR detector. The proposed CFAR detector selects an optimal CFAR detector depending on the environment. Computer simulations validate the mathematical analysis and robustness of the detector in homogeneous and nonhomogeneous backgrounds.

\section{Introduction}

The signal received in sonar or radar systems includes not only the target signal but also noise and clutter signals. The constant false alarm rate (CFAR) is a detector that maximizes the detection performance while maintaining a constant false alarm rate in noise and clutter environments. The CFAR decides the threshold adaptively according to background environments.

Figure 1 shows the typical block diagram of the CFAR. Conventional CFAR detectors adopt only a single CFAR processor, as shown in Figure 1. In this case, however, the detection performance fluctuates according to the background environment [1-3]. The cell averaging (CA) CFAR detector shows the best performance in homogeneous environments. On the other hand, its performance is poorer in nonhomogeneous environments compared to other CFAR detectors because of high threshold due to interfering signals. Some CFAR detectors, such as the smallest of (SO), greatest of (GO), and ordered statistics (OS) detectors, are used in nonhomogeneous environments. These detectors, however, are not good in homogeneous environments compared to the CA CFAR detector [4]. The excision CFAR detector was also proposed to improve the detection performance in nonhomogeneous environments [5]. In the excision CFAR detector, the total noise power was estimated by averaging the reference range cells except the cells whose powers are over a threshold. The performance is good in nonhomogeneous environments. On the other hand, its performance can be degraded over the CA CFAR detector in homogeneous environments. To solve these problems, the detector which has a good detection performance regardless of environments is needed.

The various CFAR detectors which are adopted to environments were proposed [6-10]. Among these adaptive detectors, the switching OS (SOS) CFAR detector was proposed [11]. The SOS CFAR detector improves the detection performance but still has detection loss in homogeneous environment compared with that of the CA CFAR detector.

This paper proposes an environmental adaptive (EA) CFAR detector to achieve the best performance regardless of the background environments. The proposed CFAR detector estimates the background conditions of the homogeneous and nonhomogeneous environments by comparing the power levels of the adjacent reference cells. The best CFAR detectors are used depending on the environment. Therefore, the detection performance can be maximized in any environment. Due to maximization of the detection performance, the EA CFAR can resolve the detection loss of the SOS CFAR in homogeneous environment. The proposed CFAR algorithm is also analyzed mathematically, and the validity of the mathematical analysis is proved using computer simulations. 


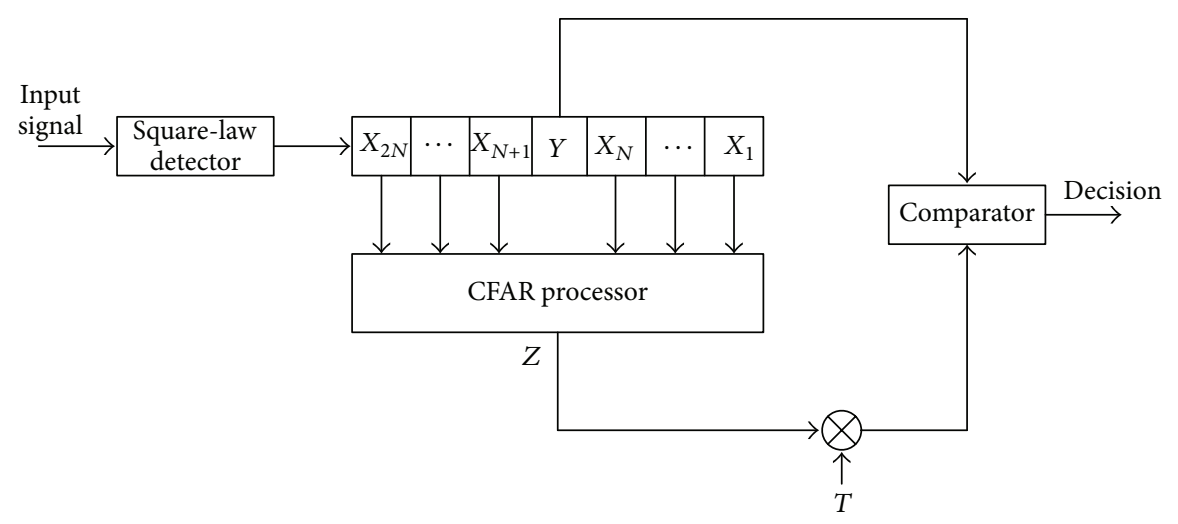

Figure 1: Block diagram of typical CFAR detector.

Conventional CFAR detectors are introduced in Section 2. We consider conventional detectors of CA CFAR and OS CFAR. And the EA CFAR is proposed and analyzed in Section 3. Section 4 describes the simulation results. The theoretical result is compared with the simulation results. The detection performance of proposed EA CFAR is also compared with those of CA CFAR and OS CFAR. The conclusions are reported in Section 5.

\section{Conventional CFAR Detectors}

2.1. Basic Model Description. In radar or sonar systems, the received signal $x(t)$ in the cells can be expressed as follows [4]:

$$
x(t)=s(t)+n(t)
$$

where $s(t)$ is the target signal and $n(t)$ is a white Gaussian noise with a zero mean $[12,13]$. Only a noise signal is observed if there is no target in the cell under test. The square-law detected output was assumed to be distributed exponentially with the probability density function (pdf) of [14]

$$
f_{X}(x)=\frac{1}{(2 \lambda)} \exp \left(-\frac{x}{2 \lambda}\right), \quad x \geq 0,
$$

where $\lambda$ is the total power. Under the null hypothesis, $H_{0}$, of no target in a range cell, the parameter, $\lambda$, is the total background clutter-plus-noise power, which is denoted as $\mu$. Under $H_{1}$ in the presence of a target, $\lambda$ is $\mu(1+S)$, where $S$ is the average signal-to-noise ratio (SNR) of a target. Therefore, $\lambda$ of the cell under test in (2) is

$$
\lambda= \begin{cases}\mu, & \text { under } H_{0}, \\ \mu(1+S), & \text { under } H_{1} .\end{cases}
$$

This paper analyzes the detection performance of CFAR detectors in the case of $\mu=1 / 2$ without loss of generality. The detection probability of a CFAR detector can be expressed with the conditional probability of

$$
P_{d}=P\left[Y>T Z \mid H_{1}\right]
$$

where $Y$ is the cell under test, $T$ is the scaling factor that maintains a constant false alarm rate, and $Z$ is the estimated background noise level that is determined by the CFAR detector.

2.2. CA CFAR Detector. The CA CFAR detector determines the threshold by adding all signal powers in the reference cells around the test cell. Figure 2 shows the CA CFAR detector, where the background power level is

$$
Z=\sum_{i=1}^{2 N} X_{i}
$$

where $X_{i}$ are the signal in the reference cells surrounding the cell under test. The CFAR condition can be obtained by multiplying the scale factor, $T$, to the estimated background level. According to (2) and (4), the detection probability of the CA CFAR detector can be expressed as [4]

$$
P_{d}=\left(1+\frac{T}{1+S}\right)^{-2 N} \text {. }
$$

The detection probability of the CA CFAR detector is the best in the homogeneous environment in which the background conditions follow the same pdf. On the other hand, the detection performance is degraded severely when the cell pdfs are different. The reason is that the threshold is increased due to interfering signals.

2.3. OS CFAR Detector. The threshold of the OS CFAR detector is obtained from one of the ordered samples of the reference cells [15]. Figure 3 shows the OS CFAR detector. And its threshold is

$$
Z=X_{(k)}
$$

where $X_{(k)}$ is the $k$ th largest sample of the reference cells. The detection probability of OS CFAR can be expressed as

$$
P_{d}=\prod_{i=0}^{k-1} \frac{2 N-i}{2 N-i+T /(1+S)} .
$$




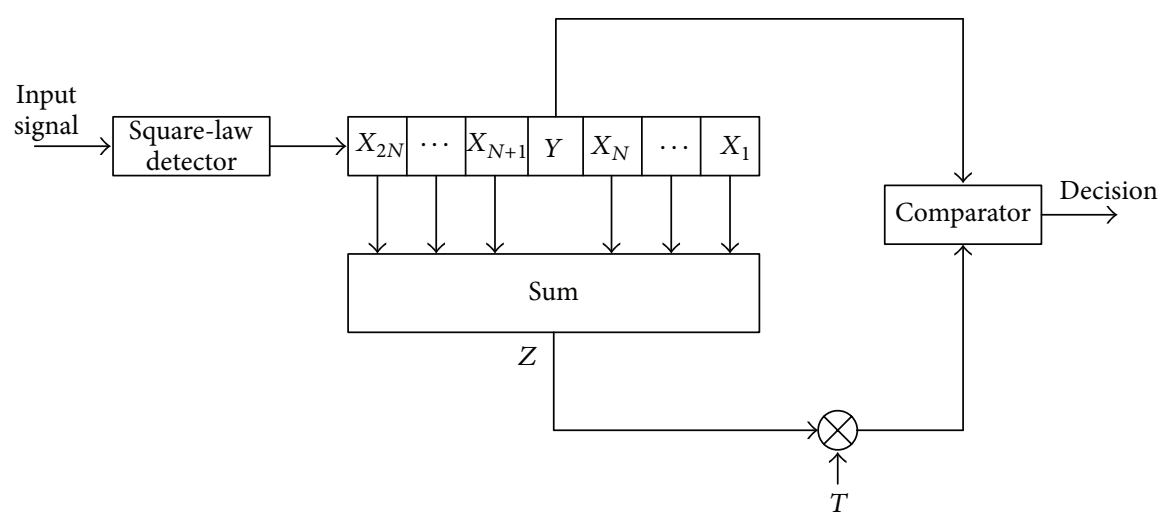

FiguRE 2: Block diagram of CA CFAR detector.

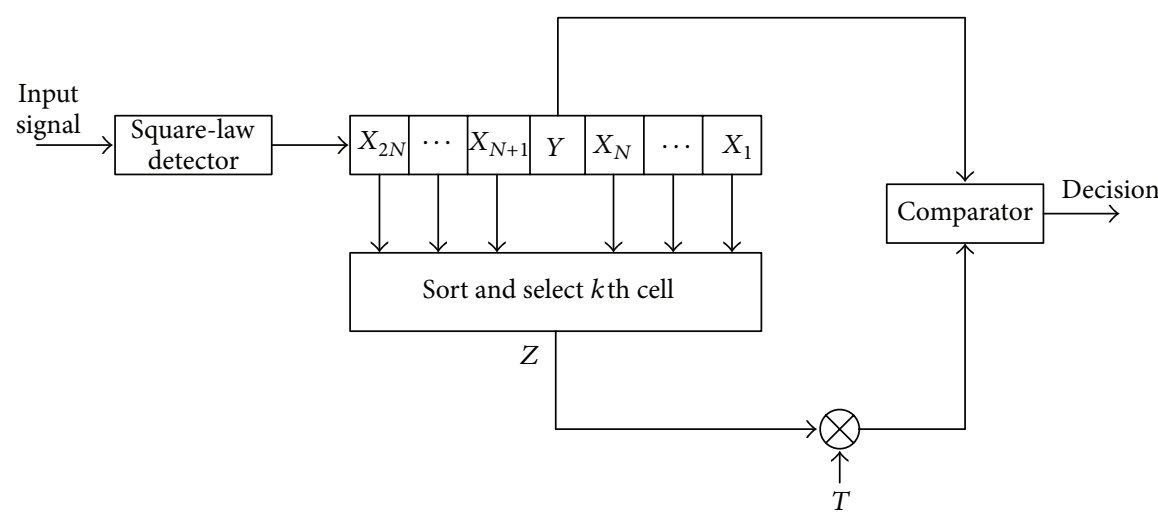

FIGURE 3: Block diagram of OS CFAR detector.

The detection probability of the OS CFAR detector varies with $k$ [15].

The OS CFAR detector compensates for defects of the CA CFAR detector in a nonhomogeneous environment. On the other hand, its performance in a homogeneous environment is poorer than that of the CA CFAR detector.

2.4. Switching OS CFAR Detector. The switching OS (SOS) detector was proposed for improving the performance of single CFAR detectors at nonhomogeneous environments [11]. The SOS CFAR detector first divides reference cells into two groups of $S_{0}$ and $S_{1}$. These groups are obtained by comparing reference cells with the scaled $Y$ of $\alpha Y$ for $\alpha<1$. If $X_{i}$ is less than $\alpha Y$, the $X_{i}$ is classified as group $S_{0}$. Otherwise, it is classified as group $S_{1}$. Next, the SOS CFAR detector estimates the background noise level based on $S_{0}$ group or whole samples of reference cells. The method that the SOS CFAR detector selects a group is based on comparing the number of samples $n_{0}$ in group $S_{0}$ with the integer threshold $N_{T}$. If $n_{0}$ is less than a threshold $N_{T}$, the background noise level will be estimated by choosing one of the ordered samples of the whole reference cells. Otherwise, the background noise level will be estimated by choosing one of the ordered samples of the samples in the $S_{0}$ group. Figure 4 represents the block diagram of SOS CFAR. The specific process of threshold selection is as follows:

$$
\begin{aligned}
& X_{i} \underset{S_{0}}{\grave{S_{1}}} \alpha Y, \quad i=1,2, \ldots, 2 N, \\
& n_{0}=\text { number of } S_{0} \text { group, }
\end{aligned}
$$$$
\text { if } n_{0}>N_{T}, \quad Z=\beta_{0} X_{\left(k_{0}\right)}, \quad \text { where } k_{0}=\operatorname{round}\left(g_{0} \times n_{0}\right)
$$$$
\text { if } n_{0} \leq N_{T}, \quad Z=\beta_{1} X_{\left(k_{1}\right)} \text {, where } k_{1}=\operatorname{round}\left(g_{1} \times 2 N\right) \text {, }
$$

where $\beta_{0}$ and $\beta_{1}$ are constant values for maintaining false alarm rate. $g_{0}$ and $g_{1}$ determine the selection number of OS CFAR detector. These are already known values; round(·) means that it rounds off the number in parenthesis.

The SOS CFAR detector selects the reference cell group according to environments. This means that the performance of the SOS CFAR detector in homogeneous environment can be worse than the CA CFAR detector.

\section{Proposed Environmental Adaptive CFAR Detector}

As mentioned in the previous section, each CFAR detector has its advantage in a specific background environment. 


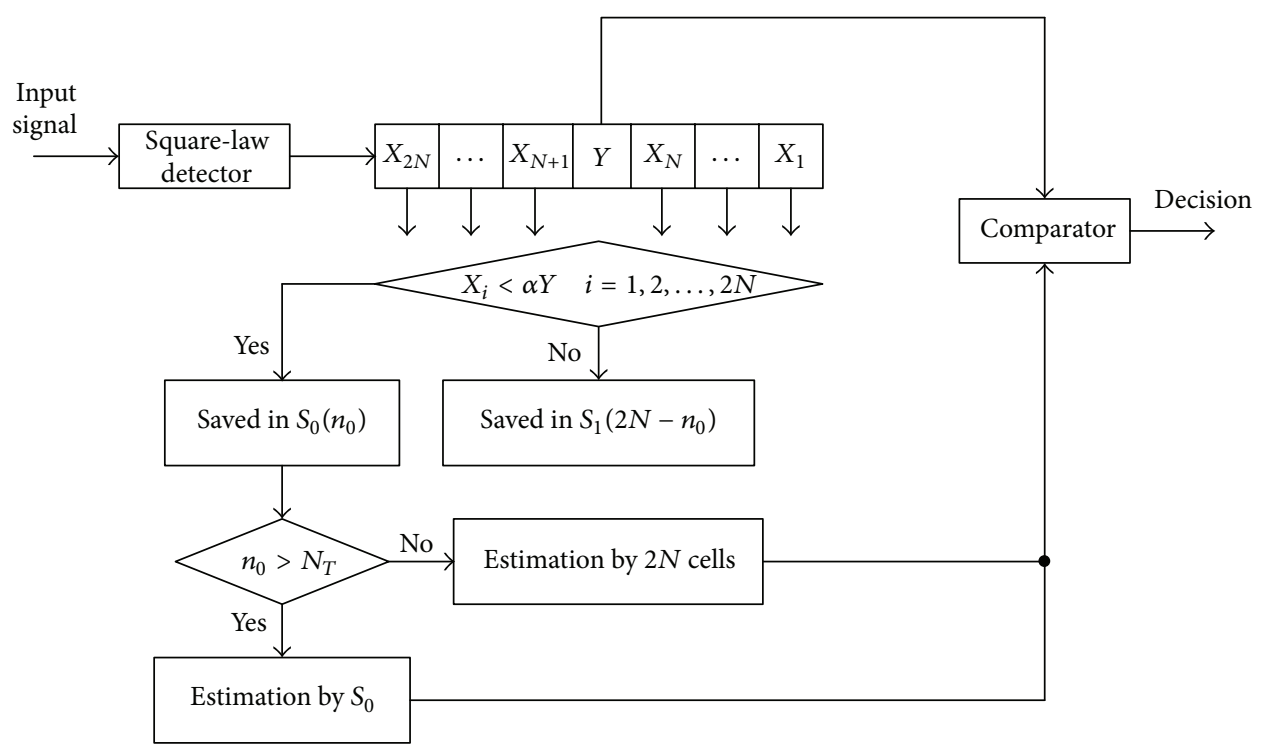

FIGURE 4: Block diagram of SOS CFAR detector.

To make a CFAR detector robust to range of background environments, this paper proposes a new CFAR detector called the EA CFAR detector. Figure 5 shows a block diagram of the EA CFAR detector. The EA CFAR first decides whether there are interfering targets and calculates the power level difference between the adjacent cells. If the difference is over a fixed threshold $C$, a flag for the cell is set to "1." Otherwise, the flag is set to "0." If the sum of all the flags is zero, the EA CFAR detector decides that the environment is homogeneous. Otherwise, the EA CFAR detector decides that the environment is nonhomogeneous.

The following were assumed when calculating the detection probability of the EA CFAR detector: (i) the samples in the cells are probabilistically independent. (ii) For the reference cells containing interfering targets, the value of $\mu$ in (3) is $\mu(1+I)$, where $I$ is the interference-to-noise ratio (INR). (iii) In multiple target environments, the interfering targets do not appear in the adjacent cells at the same time.

The detection probability of the proposed EA CFAR detector can be expressed as

$$
\begin{aligned}
P_{d}^{\mathrm{EA}}= & \left(P\left[\left|X_{k}-X_{k-1}\right|<C\right]\right)^{N} P_{d}^{\mathrm{CA}} \\
& +\left(1-\left(P\left[\left|X_{k}-X_{k-1}\right|<C\right]\right)^{N}\right) P_{d}^{\mathrm{OS}},
\end{aligned}
$$

where $P_{d}^{\mathrm{CA}}$ and $P_{d}^{\mathrm{OS}}$ are the detection probabilities of the CA and OS CFAR detectors, respectively. $N$ comparisons can be made between the powers of adjacent reference cells. If the sum of all the differences is less than a threshold $C$, the probability that the CA CFAR detector is chosen is

$$
P(\mathrm{CA})=\left(P\left[\left|X_{k}-X_{k-1}\right|<C\right]\right)^{N} .
$$

On the other hand, the OS CFAR detector is selected, whereas the EA CFAR detector considers that the background is a multiple target condition. Therefore, the probability that the OS CFAR detector is selected can be expressed as

$$
P(\mathrm{OS})=\left(1-\left(P\left[\left|X_{k}-X_{k-1}\right|<C\right]\right)^{N}\right) \text {. }
$$

The probability $P\left[\left|X_{k}-X_{k-1}\right|<C\right]$ can then be found by dividing the random variable area, as shown in Figure 6 [16]. Because $X_{k}$ and $X_{k-1}$ are independent, the joint pdf of these random variables is

$$
f_{X_{k}, X_{k-1}}\left(x_{k}, x_{k-1}\right)=\exp \left(-x_{k-1}\right) \frac{1}{1+I} \exp \left(-\frac{x_{k}}{1+I}\right) \text {. }
$$

In the case of area $A$, the probability $P\left[\left|X_{k}-X_{k-1}\right|<C\right]$ is as follows:

$$
\begin{aligned}
P(A)= & \int_{0}^{C} \int_{0}^{x_{k-1}+C} f_{X_{k}, X_{k-1}}\left(x_{k}, x_{k-1}\right) d x_{k} d x_{k-1} \\
= & 1-\exp (-C)-\frac{1+I}{2+I} \exp \left(-\frac{C}{1+I}\right) \\
& +\frac{1+I}{2+I} \exp \left(-\frac{(3+I) C}{1+I}\right) .
\end{aligned}
$$

In another case of area $B$, the probability $P\left[\left|X_{k}-X_{k-1}\right|<C\right]$ is as follows:

$$
\begin{aligned}
P(B) & =\int_{C}^{\infty} \int_{x_{k-1}-C}^{x_{k-1}+C} f_{X_{k}, X_{k-1}}\left(x_{k}, x_{k-1}\right) d x_{k} d x_{k-1} \\
& =\frac{1+I}{2+I} \exp (-C)-\frac{1+I}{2+I} \exp \left(-\frac{(3+I) C}{1+I}\right) .
\end{aligned}
$$

Therefore, the final selection probability is the sum of each area probability such as

$$
\begin{aligned}
& P\left[\left|X_{k}-X_{k-1}\right|<C\right] \\
& \quad=1-\frac{1}{2+I} \exp (-C)-\frac{1+I}{2+I} \exp \left(-\frac{C}{1+I}\right) .
\end{aligned}
$$




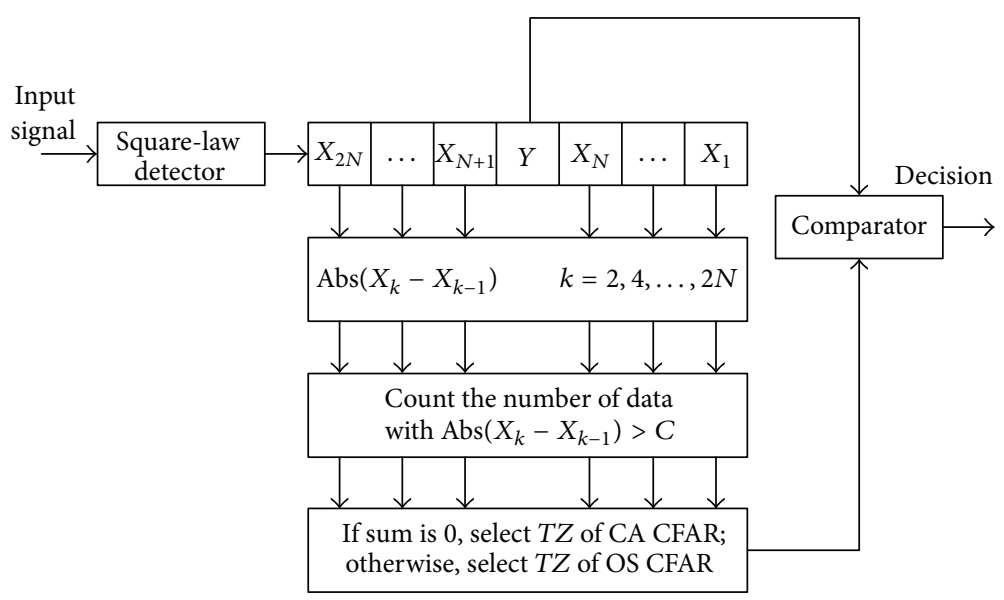

FIGURE 5: Block diagram of EA CFAR detector.

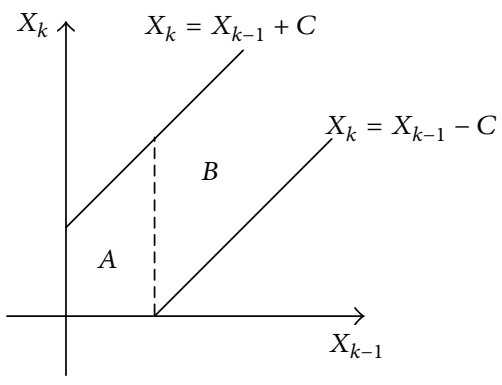

Figure 6: $P\left[\left|X_{k}-X_{k-1}\right|<C\right]=P\left[X_{k-1}-C<X_{k}<X_{k-1}+C\right]$.

Finally, the detection probability of the proposed CFAR detector can be expressed from (6), (8), (10), and (16):

$$
\begin{aligned}
P_{d}= & \left(1+\frac{T}{1+S}\right)^{-2 N} \\
& \times\left(1-\frac{1}{2+I} \exp (-C)-\frac{1+I}{2+I} \exp \left(-\frac{C}{1+I}\right)\right)^{N} \\
& +\prod_{i=0}^{k-1} \frac{2 N-i}{2 N-i+T /(1+S)} \\
& \times\left(1-\left(1-\frac{1}{2+I} \exp (-C)-\frac{1+I}{2+I} \exp \left(-\frac{C}{1+I}\right)\right)^{N}\right) .
\end{aligned}
$$

\section{Simulation Results}

To verify the performance of the proposed EA CFAR detector, it was compared with the CA, OS, and SOS CFAR detectors in homogeneous and nonhomogeneous environments. Table 1 lists the simulation parameters. For the OS CFAR detector, the value, $k$, was set to 21 because it shows the best performance [15]. To maintain a false alarm rate $10^{-6}$ in the SOS CFAR detector, $g_{0}$ and $g_{1}$ were set to 0.93 and $\beta_{0}$ and $\beta_{1}$ were set to 9.34. In addition, $\alpha$ was set to 0.9 . In Figure 7 , the mathematical analysis result is compared with

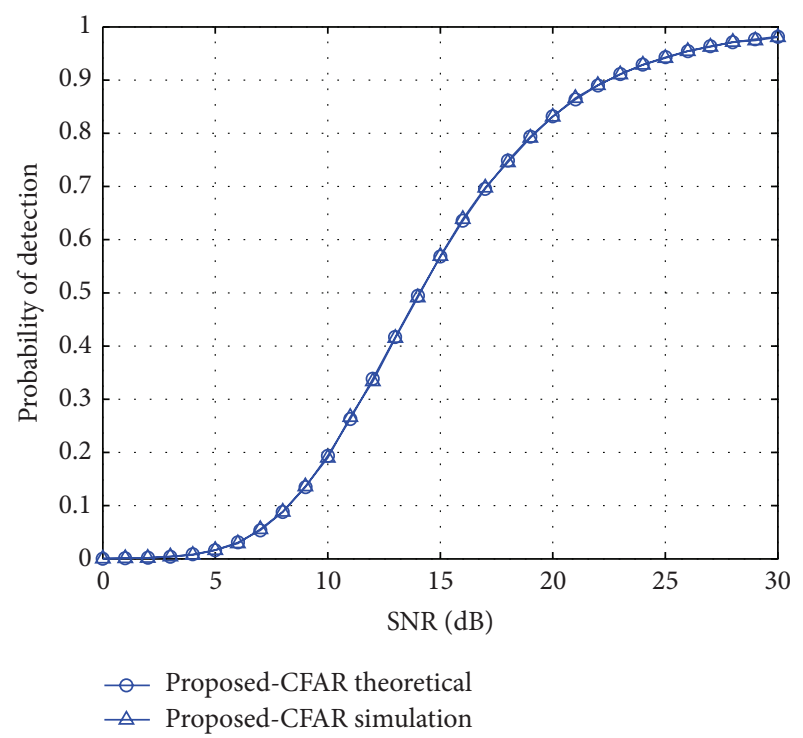

FIGURE 7: Comparison between the theoretical analysis and simulation.

TABLE 1: Common simulation parameters.

\begin{tabular}{lc}
\hline Parameters & Value \\
\hline The number of reference cells & 24 \\
Desired false alarm rate & $10^{-6}$ \\
Selection number of OS CFAR & 21 (optimum) \\
Nonhomogeneous environment & Multiple targets \\
\hline
\end{tabular}

the simulation result. Figure 7 shows that the mathematical analysis matches the simulation result. Figures 8 and 9 show the detection performance according to the threshold, $C$, at each environment. The detection probabilities vary according to the threshold $C$ and the detection probabilities increases with increasing $C$. If $C$ is over six, the detection probabilities are almost saturated. In a multiple target situation, the effect of $C$ is not critical. 


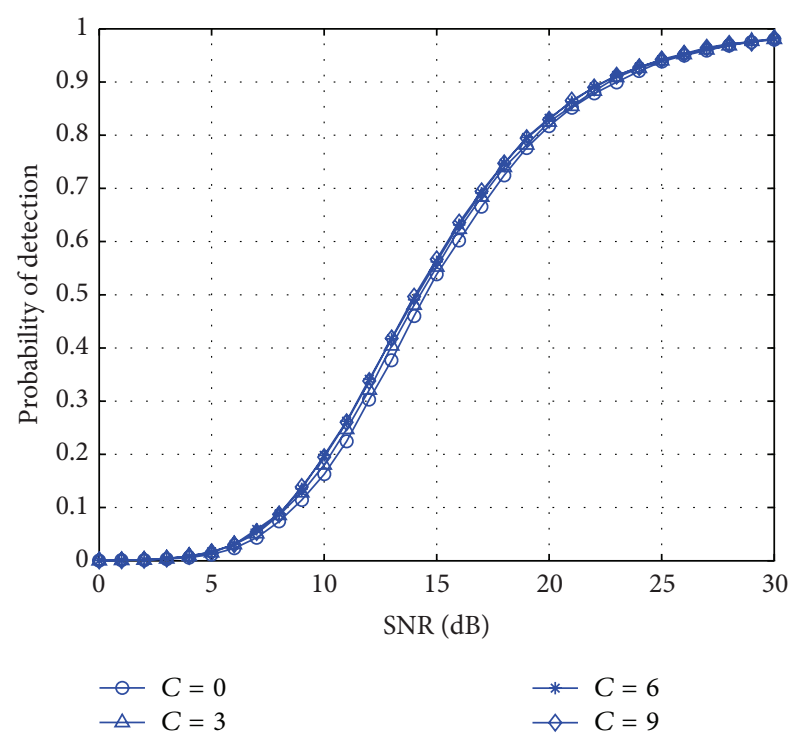

FIGURE 8: Detection performance according to the threshold $C$ at homogeneous environment.

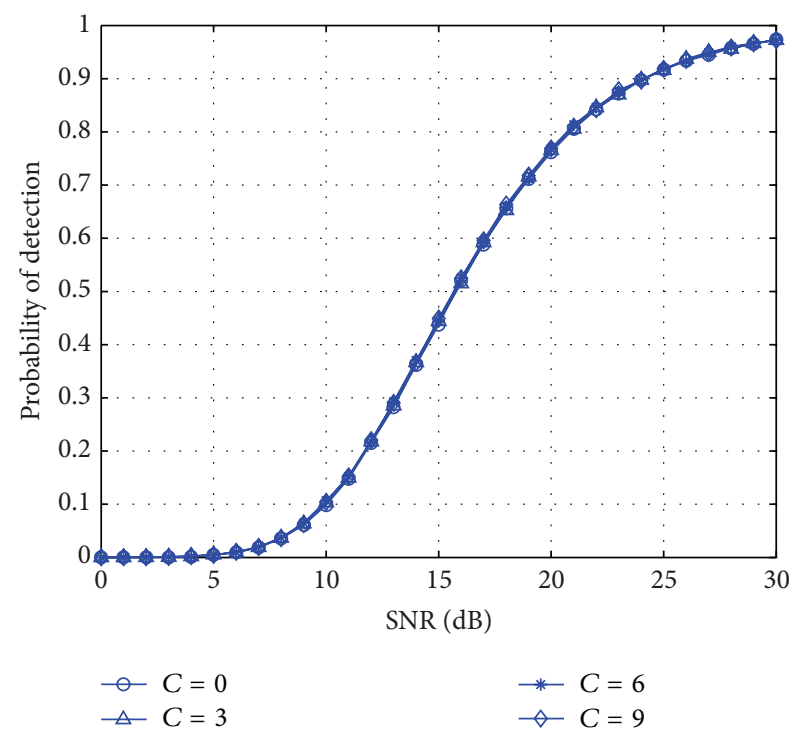

FIGURE 9: Detection performance according to the threshold $C$ at two multiple targets environment.

Figure 10 shows the detection performances of the CA CFAR, OS CFAR, SOS CFAR, and EA CFAR detectors in a homogeneous environment. $C$ was set to nine because of the best detection performance. As shown in the figure, the EA CFAR detector showed better detection performance over OS CFAR and SOS CFAR and similar detection performance to the CA CFAR. And the detection performance of the EA CFAR detector has about $1 \mathrm{~dB}$ gain compared with that of the SOS CFAR.

Figure 11 shows the detection performances of the CA, OS CFAR, SOS CFAR, and EA CFAR detectors in two interfering targets. The EA CFAR detector follows the advantage of the OS CFAR detector which is robust to interfering targets.

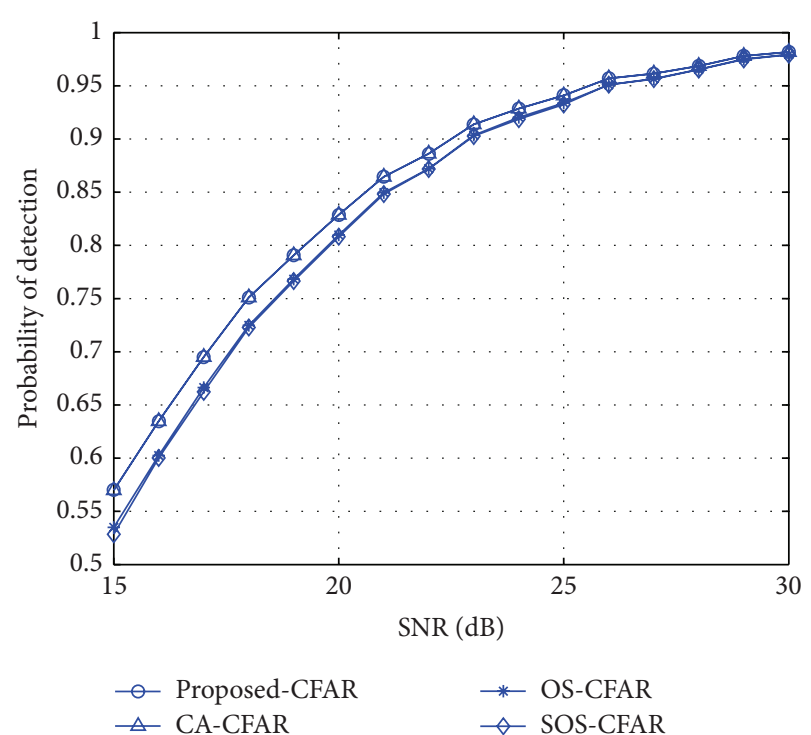

FIGURE 10: Detection performance comparison of CFAR detectors at homogeneous environment.

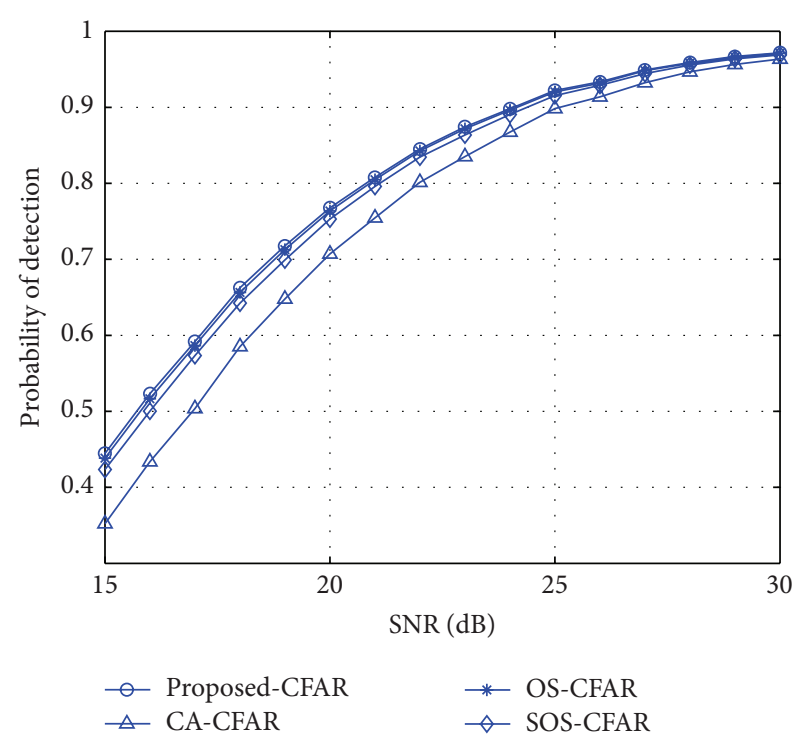

FIGURE 11: Detection performance comparison of CFAR detectors at two multiple targets environment.

According to the simulation results, the proposed EA CFAR detector shows the best performance in any environment.

\section{Conclusions}

In this paper, the EA CFAR detector was proposed and analyzed mathematically. The proposed detector estimates the environments using the difference in the adjacent reference cell powers. The EA CFAR detector then selects the optimal CFAR detector according to the background environment. A theoretical analysis of the EA CFAR detector was obtained. The proposed EA CFAR detector showed 
the best performance because it is not interrupted by the environment.

\section{Conflict of Interests}

The authors declare that there is no conflict of interests regarding the publication of this paper.

\section{Acknowledgments}

This research was supported by the MSIP (Ministry of Science, ICT \& Future Planning), Korea, under the C-ITRC (Convergence Information Technology Research Center) support program (NIPA-2014-H0401-14-1004) supervised by the NIPA (National IT Industry Promotion Agency).

\section{References}

[1] M. A. Habib, M. Barkat, B. Aissa, and T. A. Denidni, "CA-CFAR detection performance of radar targets embedded in "non centered chi-2 gamma" clutter," Progress in Electromagnetics Research, vol. 88, pp. 135-148, 2008.

[2] B. Magaz, A. Belouchrani, and M. Hamadouche, "A new adaptive linear combined CFAR detector in presence of interfering targets," Progress in Electromagnetics Research B, vol. 34, pp. 367-387, 2011.

[3] M. B. El Mashade, "Performance improvement of adaptive detection of radar target in an interference saturated environment," Progress in Electromagnetics Research M, vol. 2, pp. 57-92, 2008.

[4] P. P. Gandhi and S. A. Kassam, "Analysis of CFAR processors in homogeneous background," IEEE Transactions on Aerospace and Electronic Systems, vol. 24, no. 4, pp. 427-445, 1988.

[5] H. Goldman and I. Bar-David, "Analysis and application of the excision CFAR detector," IEE Proceedings F: Communications, Radar and Signal Processing, vol. 135, no. 6, pp. 563-575, 1988.

[6] L. Tabet and F. Soltani, "A generalized switching CFAR processor based on test cell statistics," Signal, Image and Video Processing, vol. 3, no. 3, pp. 265-273, 2009.

[7] T. T. V. Cao, "Constant false-alarm rate algorithm based on test cell information," IET Radar, Sonar \& Navigation, vol. 2, no. 3, pp. 200-213, 2008.

[8] S. Erfanian and V. T. Vakili, "Introducing excision switchingCFAR in $K$ distributed sea clutter," Signal Processing, vol. 89, no. 6, pp. 1023-1031, 2009.

[9] B. Magaz, A. Belouchrani, and M. Hamadouche, "Automatic threshold selection in OS-CFAR radar detection using information theoretic criteria," Progress in Electromagnetics Research B, no. 30, pp. 157-175, 2011.

[10] N. N. Liu, J. Li, and Y. Cui, "A new detection algorithm based on CFAR for radar image with homogeneous back-ground," Progress in Electromagnetics Research C, vol. 15, pp. 13-22, 2010.

[11] S. Erfanian and V. T. Vakili, "Introducing switching ordered statistic CFAR type I in different radar environments," EURASIP Journal on Advances in Signal Processing, vol. 2009, Article ID 525704, pp. 1-11, 2009.

[12] M. B. El Mashade, "Analysis of CFAR detection of fluctuating targets," Progress in Electromagnetics Research C, vol. 2, pp. 6594, 2008.
[13] M. B. El Mashade, "Performance analysis of OS structure of CFAR detectors in fluctuating target environments," Progress in Electromagnetics Research C, vol. 2, pp. 127-158, 2008.

[14] D.-S. Han, "Detection performance of CFAR detectors based on order statistics for partially correlated chi-square targets," IEEE Transactions on Aerospace and Electronic Systems, vol. 36, no. 4, pp. 1423-1429, 2000.

[15] H. Rohling, "Radar CFAR thresholding in clutter and multiple target situations," IEEE Transactions on Aerospace and Electronic Systems, vol. 19, no. 4, pp. 608-621, 1983.

[16] A. Leon-Garcia, Probability, Statistics, and Random Processes for Electrical Engineering, Pearson Education, New Jersey, NJ, USA, 2008. 


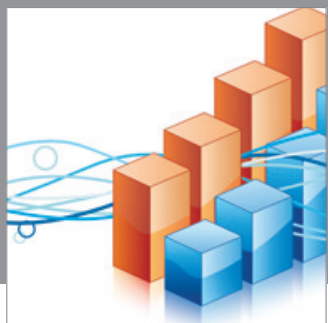

Advances in

Operations Research

mansans

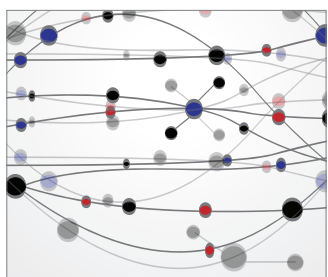

The Scientific World Journal
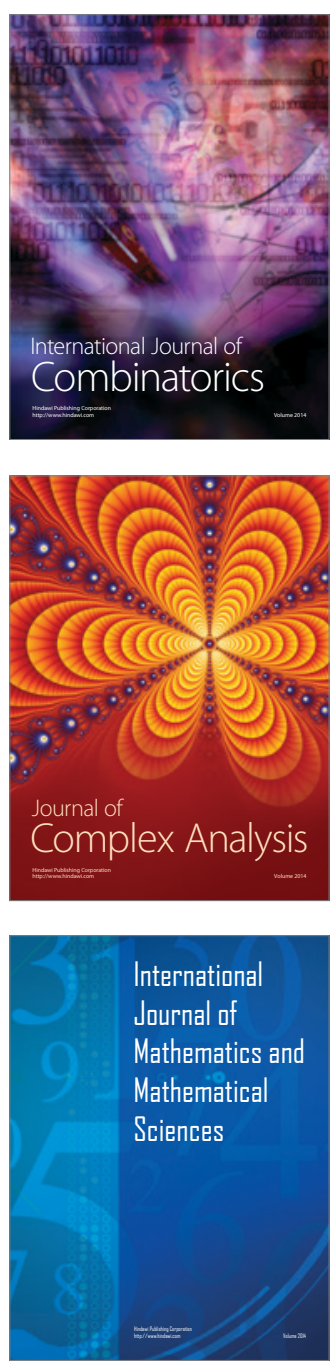
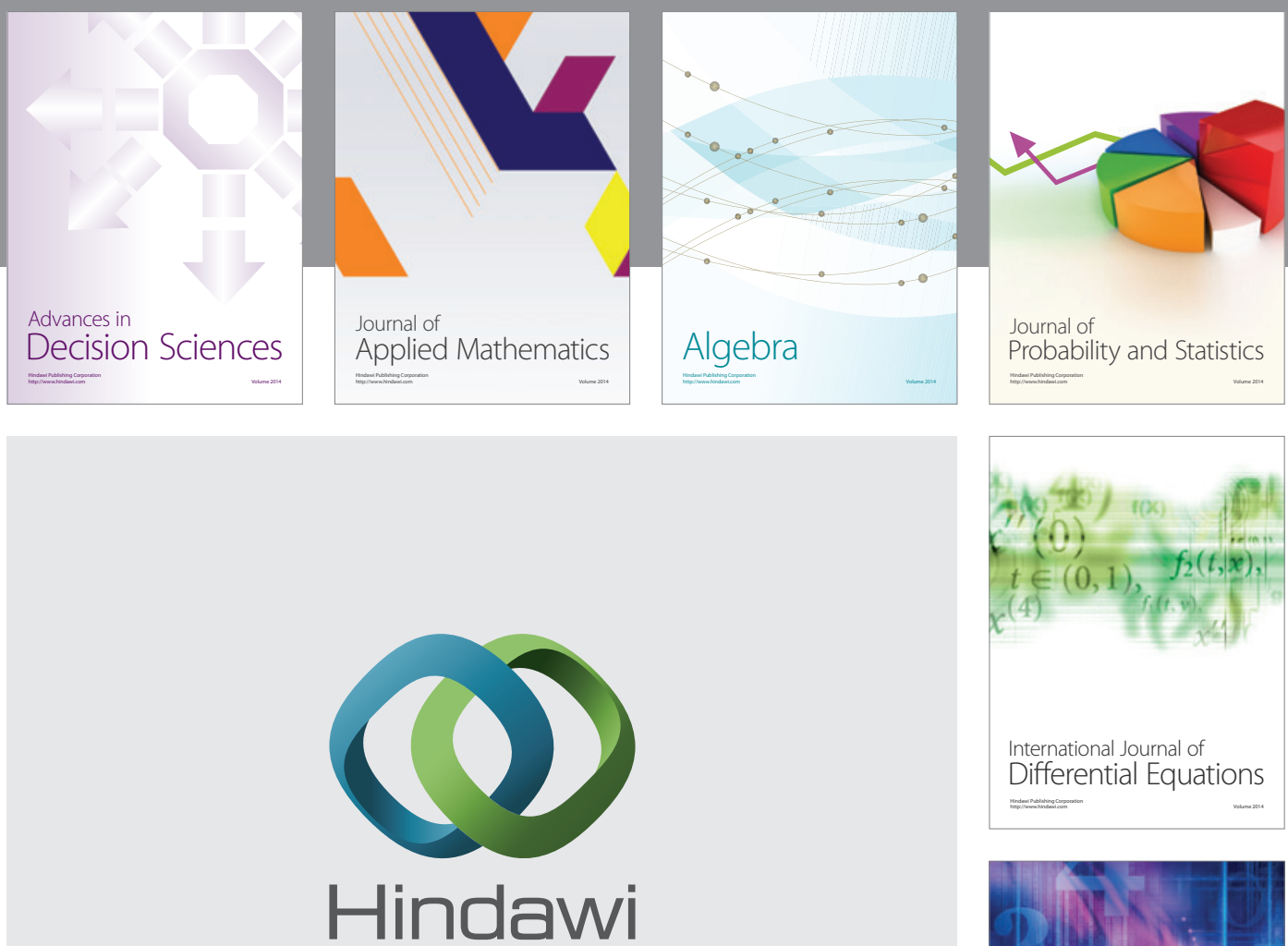

Submit your manuscripts at http://www.hindawi.com
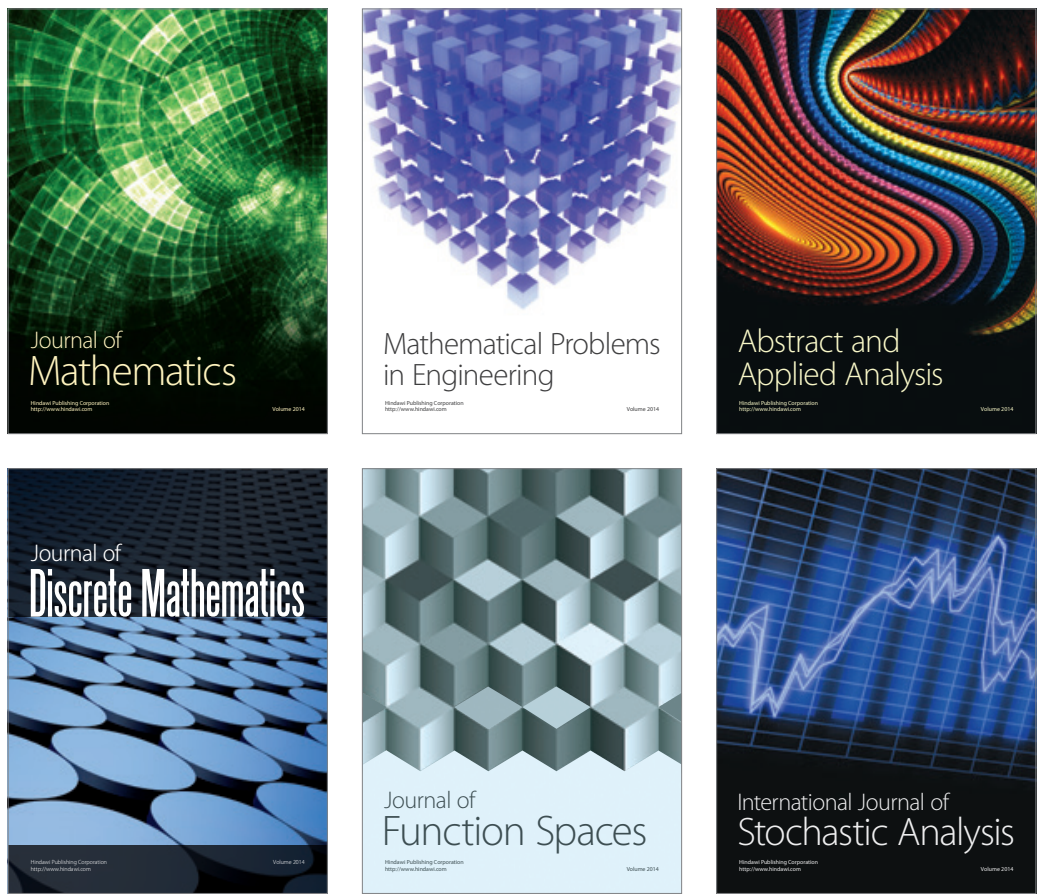

Journal of

Function Spaces

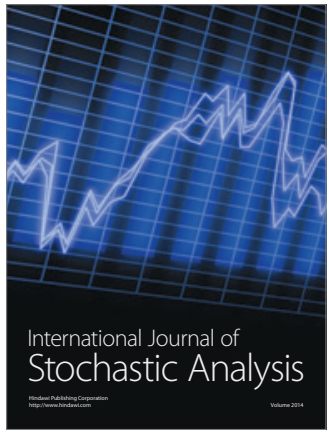

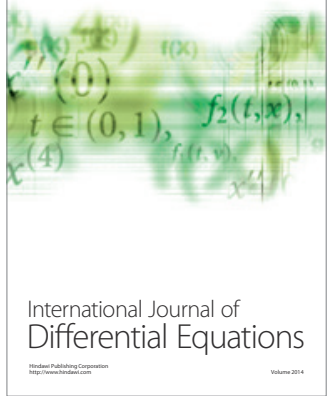
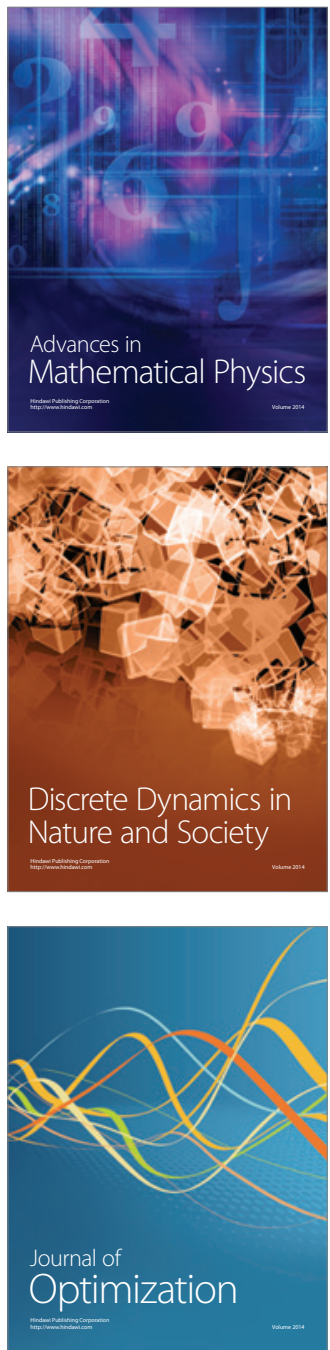